\title{
New Position of University Librarians in RFID Technology Environment Lei Wang
}

Shenyang University of Aeronautics and Astronautics

\begin{abstract}
In information age, the traditional service of library management system based on barcode and magnetic stripe has gradually shown their deficiency. Therefore, it is necessary to introduce new and high technology and change the existing service dilemma. The application of RFID technology in the university library, and the influence on the University Library and librarian are talked briefly.
\end{abstract}

Keywords: RFID; University library; Positioning

\section{RFID 技术环境下高校图书馆员的新定位}

\author{
王磊 \\ 沈阳航空航天大学
}

摘要: 信息化时代, 基于磁条和条形码技术的图书馆管理系统, 其传统服务也逐渐表现出不足。所以图书馆引进高新技术, 改变现有的服务客境显得尤为必要。简述随着 RFID 技术在高校图书馆的应用, 对高校图书馆的影响及图书馆馆员产生什么 样重新定位。

关键词: RFID、高校图书馆、定位

\section{1 引言}

RFID 是一种快速、远距离、非接触式的自动识别技术，RFID 技术是图书馆继条形码技术后又一先进 技术的应用。这项技术优于条形码技术地方：做到图书自动盘点、图书自助借还、图书区域定位、图书自 动分拣，能够极大的提高图书馆的管理与服务水平，实现对图书的更有效、更及时的管理和控制。

在 RFID 无线射频识别技术的帮助下，图书的自助借还、高速盘点、快速查找、定位、顺架、分拣成 为了现实 $[4]$ 。

图书馆作为为公众提供服务的场所，其如何让读者得到方便高效的服务，是我们最重要的追求。目前 我校读者可以实现数字方式查询文献信息, 但真正借阅过程却依然是传统的手工方式。引进并应用 RFID 技术将会给图书馆服务带来改变, 也为图书馆事业发展赋予了更多的创造空间。应用 RFID 系统后, 带来 的首要成果是方便了广大读者。自助借还实现了多本同时借还, 借还效率大为提高, 24 小时自助还书打破 开馆时间对图书归还的限制, 极大方便了读者。自助服务同时也能对读者个人隐私的保护。在方便读者的 同时, 馆内工作效率也得到极大提高, 首先是保证了图书不被损坏, 因为 RFID 标签隐蔽性比较强, 降低了 人为破坏的几率, 另外, 大量的读者自助操作和系统智能提示降低工作人员劳动强度。最后, 以往繁重的 盘点工作变得快速、准确。

RFID 技术不仅在图书馆科技技术应用带来了革新, 也为图书馆管理观念带来更新。新的技术的应用使 许多分离的工作实现了功能上的整合与重组, 人员需求产生了变化, 人员需要重新定位, 从而改变原有人 员配置，因此图书馆必然会面临人员的重组，整合业务等问题。 


\section{2 高校图书馆人员重新定位的必要性}

\section{1 环境变化是人员重新定位的根本原因}

高校图书馆作为社会知识拥有者与传播者, 其生存与发展受社会大环境的影响。20 世纪 90 年代以来, 整个社会朝着数字化、网络化和智能化的方向快速迈进。与社会息息相关的高校图书馆自动化程度不断加 强, 一些常规的业务正在消失, 新业务又迫切的需要解决 [3]; 图书馆要想适应外部环境的变化, 适应现 代技术的挑战, 就要改革组织机构, 人员进行重新定位。

\section{$2.2 \mathrm{RFID}$ 技术在高校图书馆的应用导致人员重新定位}

\section{2.1 流通和期刊部的 “死亡”}

一直以来, 在国内高校图书管理系统中普遍采用 “安全磁条十条形码” 的技术手段, 以安全磁条作为 图书的安全保证, 以条形码作为图书的身份证, 解决了图书管理中的一些问题, 但是顺架、排架困难, 劳 动强度高, 图书查找、馆藏清点繁琐耗时, 自动化程度低, 管理缺乏人性化, 仍是急需解决的问题。RFID 技术的自动盘点、图书自助借还、图书区域定位、图书自动分拣等系统，解决图书馆工作效率低的问题， 同时提高了服务水平，传统图书馆中最主要的工作一一图书流通工作将会 “死亡”。另外, RFID 系统在不 需要人力的情况下能够对期刊进行有效管理。通过对 RFID 电子标签的设置, 读者只能在馆内阅览期刊, 而不能带出馆外。期刊部也将淡出图书馆的日常工作。

\subsection{2 新部门的设立和人力资源的重新分配}

条形码时代, 高校图书馆采用柜台式借阅和馆员看守式阅览方式, 引进 RFID 技术后, 这一借阅方式 和阅览方式的消失使读者与馆员的交流机会变少。图书馆作为服务行业, 为了更好的服务, 增设新的专门 的服务部门是一种趋势; “死亡” 的流通和期刊部, 为图书馆提供了富裕的人员, 这些人力资源面对重新 分配定位的问题。

\subsection{3 图书馆服务的改变}

由 RFID 自助借还、自助还书机和 RFID 门禁系统取代了柜台式借阅方式，图书馆将不再以读者对象或 文献类型划分阅览室和书库，文献资料将尽量实行集中统一管理方式，全馆只有一个出入口，读者一次进 馆便可随意阅览所有书刊, 方便管理和节省人力。期刊和图书可以共处一室, RFID 系统对粘贴有电子标签 的期刊和图书进行管理, 期刊部、流通部、前台合并为一个读者服务部, 负责期刊、图书的归架管理工作 及读者的简单咨询工作。如向读者解答借还、阅览等一般性咨询, 为读者示范自助借还机、自助还书机的 使用方法, 向有关部门反馈读者的意见和要求, 提高图书馆的服务质量同时也将人力资源从重复劳动中转 移到更有价值的工作中。RFID 图书馆在服务效率上、形式和内容发生了变化, 从而引发图书馆业务服务的 改变。

\section{3 人员新定位的方法}

每个行业, 岗位设定以及人员分配都是以本行业的业务流程为准则而进行设计。RFID 技术应用于图书 馆, 原来传统的业务流程及人员设定已经不再适合。岗位重新设定, 人员重新分配。有效提高图书馆的整 体服务水平。使图书馆的工作真正做到 “读者第一, 服务至上”。

\section{1 部门的重新规划}

以沈阳航空航天大学为例 (以下的文章中简称我校), 我校图书馆现有在职职工 53 人, 原有部门设置: 
办公室, 技术部, 参考咨询部, 采编部, 流通部, 期刊部; 实施 RFID 技术后, 新的部门设置: 办公室, 技术部，信息部，采编部，服务台。原有的流通部和期刊部消失，设置总服务台处理日常读者借还书过程 遇到的一些问题; 同时把读者意见和要求反馈有关部门。使图书馆的服务更加人性化。

\section{2 人员的重新分配}

3.2 .1 我校图书馆人员的基本情况（截止本文撰写时):

表 1 岗位设置详表（人）

\begin{tabular}{|l|l|l|l|l|l|l|}
\hline 总人数 & 流通部 & 期刊部 & 办公室 & 技术部 & 参考咨询部 & 采编部 \\
\hline 53 & 26 & 7 & 4 & 8 & 3 & 5 \\
\hline
\end{tabular}

表 2 在职人员学历情况（人）

\begin{tabular}{|l|l|l|l|l|}
\hline 总人数 & 研究生 & 本科生 & 专科生 & 专科以下 \\
\hline 53 & 9 & 23 & 5 & 6 \\
\hline
\end{tabular}

表 3 职称情况（人）

\begin{tabular}{|l|l|l|l|l|}
\hline 总人数 & 高级职称 & 副高 & 中级职称 & 初级以下 \\
\hline 53 & 1 & 6 & 16 & 30 \\
\hline
\end{tabular}

从本人调查情况来看，我校图书馆有 11 人毕业于图书情报专业。其他人员都是转行从事这一职业。 专业素质水平较低, 职称相对来说较低, 初级职称以下人员占约 $60 \%$ 。在条形码时代, 图书馆馆员每天忙 于借书、还书、上架、整架。没时间钻研业务、学习新知识; 服务水平不能与时代同步。

\section{2 .2 人员重新定位的实施办法：}

自动化、现代化技术在图书馆的应用，原来一些比较重要部门没有存在的必要而消失，节省大部分人 员; 而以前不太重要的部门变成了比较重要的部门; 如技术部、采编部、参考咨询部等。人员的重新定 位已经是势在必行。

1. 对全馆人员进行 RFID 技术在图书馆的应用培训:

RFID 技术应用与图书馆也带来图书馆在服务领域的革新。新系统的引入对图书馆员也是一个新的挑 战。图书馆对全体馆员进行业务培训, 让每一个馆员都应掌握 RFID 技术的工作原理和 RFID 技术各个系统 的使用方法，学习新的馆藏文献的位置和分布情况，掌握每楼层的馆藏文献分布情况。同时根据本馆的实 际情况对人员重新调整。

\section{2. 加强技术服务部队伍的建设}

技术服务部的工作与其他各个部门紧密相关，随着图书馆自动化程度的不断提升，技术服务部的工作 量也在不断增加。RFID 技术的采用加强了技术服务部的关键地位, 它不仅担负着整个图书馆计算机软硬件 的维护、网络的应用和优化，而且还要保证 RFID 硬件设施、RFID 管理系统的正常运行。

我馆技术部现有 8 名馆员，有 5 名馆员负责电子阅览室的日常工作，剩余 3 名馆员负责数字资源的正 常使用, 网站的正常运行，图书馆自动化系统的正常运行。办公机、检索机、服务器的正常运行。[1]实 施 RFID 系统以后，技术部在原有的工作以外，增加精通网络和编程的研发人员，RFID 技术完全不同于图 
书馆自动化系统, 不但要与图书馆行业紧密结合, 符合图书馆管理与服务的普遍需要, 也要与具体的图书 馆发生关联，与图书馆的应用系统有机整合，在具体的场地、环境中 “量身订做”。可以说，RFID 技术的 应用是一个复杂的、个性化的应用系统工程不是简单的设备或软件引进。需要图书馆全面参与技术引进过 程, 调整业务流程, 改进原有系统, 因此我馆从现有人员中挑选有关计算机专业人员充实到技术部的队伍 里，以切实提高管理与服务。

\section{3. 加强采编队伍的建设}

采访编目是图书馆工作的基础，担负着图书的查重、采购、分类、著录、目录组织。实施 RFID 后，， 图书馆 RFID 文献管理系统应用所依靠的书目数据、文献载体都由采编部门完成, 对采编质量提出了更严 格的要求, 采编部门增加了许多新的业务工作, 图书粘贴电子标签、电子标签上号, 将其输入 RFID 图书 管理系统, 同时采编部也要对期刊做同样的工作, 实施 RFID 系统以后实行的是大开架阅览, 新书的加工 量加大, 5 名采编人员 (其中 2 名采访人员和 3 名编目人员) 明显偏少, 需要对 RFID 系统应用后节省下来的 文献借阅工作人员进行培训，让他们从事编目工作。

\section{4. 加强信息队伍的建设}

截止目前, 我校图书馆信息部有 3 人。真正高效率、高质量地服务, 仅靠 3 人的知识和精力可谓势单 力薄。信息部所要完成的工作如下: 一是提供读者培训服务, 文献信息检索课讲授; 二是文献传递、学科 服务和馆际互借; 三是参考咨询服务。目前, 我校参考咨询服务以非实时, 和实时服务为主, 主要采用读 者留言、FAQ、QQ、参考咨询 4 种方式。读者留言是由读者在图书网页留言板块中留言, 由负责人员负责 解答; 常见问题库 $(\mathrm{FAQ})$ 是对读者利用图书馆时的常见问题进行解答; $Q Q$ 为实时在线为读者回复问题; 参 考咨询是通过电子邮件方式传递; 读者在登录后在 “我要提问”一栏中提交自己的问题，参考咨询员登录 后台及时回复给读者。

\section{4 图书馆人员定位与展望}

图书馆业务发展的重中之重还是对未来图书馆人员正确的定位，人尽其用。网络环境下，读者的信息 需求呈现个性化发展的趋势，图书馆传统的信息服务模式已经不能满足读者的个性化需求。

RFID 系统的引进使传统图书馆的图书借还工作淡出了人们的视野。节省的一部分人力资源, 图书馆依 据本馆工作人员的专业特点, 组成一支专业队伍, 实现图书馆信息资源和信息中介的价值。

如高校图书馆作为重要的学术交流机构之一,传统的信息收集、组织、传播以及保存的服务角色已不 能完全适应当前的学术交流环境, 也难以满足科研用户多元且复杂的科研需求。新的学术交流环境下, 高校 图书馆的角色转型与创新成为保持其学术交流机构活力的重要趋势及方向。

未来图书馆馆员改变服务观念, 重新定位, 向更专业, 更加技术的专门人才发展, 面临角色转型也面 临工作重心转移。

\section{5 结束语:}

RFID 技术使图书馆管理进入自动化时期, 导致图书馆业务流程和管理技术发生变革，迫使图书馆馆员 服务观念发生改变, 岗位设置随之发生了变化。科技历来以人为本, 科技变革方便读者, 减轻无必要的体 力劳动强度, 从而有更多的精力去提高图书馆的服务水平和服务质量, 让图书馆业务更加深入的发展, 为 读者服务。 


\section{参考文献:}

[1] 韩芬 浅论 RFID 技术应用后图书馆员角色的转换 [科技情报与经济 $] 2012$

[2] 赵晓芳 基于 RFID 的图书馆编目业务重组与发展[图书与情报] 2011

[3] 刘传筀 周秀会 董真 基于 RFI D 技术的图书馆业务流程重组 [图书馆论坛] 2008

[4] 撖立军 RFID 技术在图书馆的应用现状与问题分析 [电子设计工程]

[5] 周春玲 图书馆个性化服务的几个新看点[图书馆学刊 2009

[6] 王海树 我国图书馆 RFID 技术应用研究--以安徽大学图书馆 RFID 项目建设为例 [安徽大学] 2014

\section{References:}

[1] Han Fen. Brief Study on the Role of Librarians after the Application of RFID Technology [Science and Technology Information and Economy] 2012

[2] Zhao Xiaofang Library Catalogue Reorganization and Development Based On RFID [Book and Information] 2011

[3] Liu Chuanxi, Zhou Xiuhui, Dong Zhen. Library Business Process Reengineering Based on RFI D Technology [Library Tribune] 2008

[4] Han Lijun. Application Status and Problem Analysis RFID Technology in the Library [Electronic Design Engineering]

[5] Zhou Chunling. New Views of Library Personalized Services [Library Journal] 2009

[6] Wang Haishu. Study on the Application of RFID Technology in China's Library - Taking Anhui University Library RFID Project Construction as an Example [Anhui University] 2014 